Postprint: Tijskens A., Roels S., Janssen H., 2019. Neural networks for metamodelling the hygrothermal behaviour of building components. Building and Environment 162: 106282

\title{
Neural networks for metamodelling the hygrothermal behaviour of building components
}

\author{
Astrid Tijskens $^{a, *}$, Staf Roels ${ }^{a}$, Hans Janssen ${ }^{a}$ \\ ${ }^{a}$ KU Leuven, Department of Civil Engineering, Building Physics Section, Kasteelpark Arenberg 40, 3001 Leuven, Belgium \\ * Corresponding author. Tel.: +32 16 323808; E-mail address: astrid.tijskens@kuleuven.be
}

\section{ABSTRACT}

When simulating the hygrothermal behaviour of a building component, there are many inherently uncertain parameters. A probabilistic evaluation takes these uncertainties into account, allowing a more dependable assessment of the hygrothermal behaviour. However, this often necessitates many Monte Carlo simulations, which easily become computationally inhibitive. To overcome this time-expense problem, the hygrothermal model can be replaced by a metamodel, a much simpler mathematical model which aims at mimicking the original model with a strongly reduced calculation time. In this paper, a metamodel is developed to directly predict hygrothermal time series (e.g. temperature, relative humidity, moisture content), rather than singlevalued derived performance indicators (e.g. maximum mould index), as these hygrothermal time series yield more information, and also allow the user to post-process the output as desired. So far, no metamodelling strategies able to tackle time series are available in the field of building physics. Because the hygrothermal response of a building component is highly non-linear and transient, this paper focuses on neural networks for time series, as they have proven successful in many other fields. The performance and training time of three popular types of networks (multilayer perceptron, recurrent neural network, convolutional neural network) is evaluated based on an application example of a massive masonry wall. The results indicate that only the recurrent and convolutional networks are able to capture the complex patterns of the hygrothermal response. Additionally, the convolutional network performed significantly better and was 10 times faster to train for the current application example, compared to the recurrent network.

\section{KEYWORDS}

Metamodelling; Neural networks, Time series modelling; Probabilistic assessment; Hygrothermal assessment 
Postprint: Tijskens A., Roels S., Janssen H., 2019. Neural networks for metamodelling the hygrothermal behaviour of building components. Building and Environment 162: 106282

https://doi.org/10.1016/j.buildenv.2019.106282

\section{Introduction}

In recent years, traditional deterministic assessments in building physics have evolved towards a probabilistic framework [1-3]. When simulating the hygrothermal behaviour of a building component, there are many inherently uncertain parameters, such as the exterior and interior climate. When evaluating existing walls, even the exact component geometry and material properties are often unknown. A deterministic assessment often does not suffice to come to a reliable design decision or conclusion, whereas a probabilistic evaluation includes these uncertainties, and thus allows assessing the hygrothermal behaviour and the related damage risks in a more reliable manner. However, the thereto frequently adopted Monte Carlo approach [4] often involves thousands of simulations, and therefore easily becomes computationally inhibitive. To overcome this time-expense problem, the hygrothermal model can be replaced by a metamodel, a much simpler mathematical model which mimics the original model with a strongly reduced calculation time. Often, when one desires to predict a single-valued output based on single-valued inputs, simple metamodels suffice. For example, in Van Gelder et al. [5], different metamodelling strategies are used to predict the cumulative heat demand and the number of hours with temperatures exceeding $25^{\circ} \mathrm{C}$ of a semi-detached dwelling, based on the probabilistic input variables. In that work, polynomial regression, multivariate adaptive regression splines, kriging, radial basis function networks and neural networks (i.e. multilayer perceptron) are applied. In another example, a statistical metamodel is constructed to predict the moisture risk of internal wall insulation [6]. That moisture risk was quantified via a single-valued response (maximum relative humidity or maximum mould index) and generalised additive models were used to model the relation between the input variables and the response value.

The main disadvantage of the approach in these examples is that these metamodels are constructed for a specific and single-valued performance indicator (e.g. the cumulative heat demand or the maximum mould index). If the user wishes to evaluate performance using a different indicator (e.g. the peak heating demand or the average mould index), a new metamodel needs to be constructed, which is time intensive. Also, single- 
Postprint: Tijskens A., Roels S., Janssen H., 2019. Neural networks for metamodelling the hygrothermal behaviour of building components. Building and Environment 162: 106282

https://doi.org/10.1016/j.buildenv.2019.106282

valued performance indicators might not provide sufficient information for decision-making. For example, the maximum mould index, which is based on the temperature and relative humidity time series, only reveals the maximum value during a given period, but does not give insight in how long or how often this maximum occurs, and neither does it provide any indication on the mould index for the rest of the time. Instead, a metamodel predicting hygrothermal output time series, as calculated by the original hygrothermal model, provides more flexibility, as it provides more information and allows the user to post-process the output as desired. However, the relations between input time series (e.g. exterior climate, interior climate) and output time series (e.g. temperature, relative humidity, moisture content ...) are much more complex, because the hygrothermal response of a building component is highly non-linear and transient. The metamodel must thus be able to capture these complex patterns and therefore, the strategies used in the examples mentioned above no longer suffice. To the authors' knowledge, there are currently no metamodelling results available in the field of building physics that are able to predict such time series. In many other fields however, artificial neural networks have been used successfully for modelling complex and non-linear time series or sequences, from natural language modelling [7-10] over audio generation [11,12] and image and video recognition [1316] to financial predictions [17-19]. It is clear that neural networks have large potential for the metamodeling of time series. This paper hence focuses on the application of neural networks to predict hygrothermal response time series based on climate data time series.

Section 2 gives an overview of different neural network architectures and their application possibilities and (dis)advantages. In section 3, these neural networks are applied to a case study. First, the calculation object is described, after which the methodology to develop predicting neural networks is presented. Finally, the results are shown and discussed. In conclusion, the main findings are summarised and some remarks are drawn. 
Postprint: Tijskens A., Roels S., Janssen H., 2019. Neural networks for metamodelling the hygrothermal behaviour of building components. Building and Environment 162: 106282

https://doi.org/10.1016/j.buildenv.2019.106282

\section{Neural networks}

Artificial neural networks (ANN) are information-processing systems, inspired by the biological brain. The basic structure of an ANN is a network of small processing units or nodes, which represent the neurons, joined to each other by weighted connections representing the synapses between the neurons. Before an ANN can be used to make predictions, it needs to be 'trained'. By presenting the ANN input-output pairs of a training dataset, the network fits its parameters to this data, and thus learns to predict the correct output. Many varieties of ANNs have been developed over the years, with widely varying properties. The simplest neural network architecture is the multilayer perceptron (MLP). It has a feedforward architecture, meaning that its neurons are only connected from one layer to the next. Recurrent neural networks (RNN) have cyclic connections between neurons as well. This second type of networks is particularly interesting when dealing with time series or sequences $[7,8]$, as the cyclic connections allow the processing of time series. A third popular type of ANN is the convolutional neural network (CNN), often used for image processing $[13,16]$ and more recently also for sequence learning $[11,17,20]$. The characteristics of these three neural networks types are discussed in detail in the following paragraphs and subsequently applied to predict the hygrothermal response of a massive masonry wall in section 3.

\subsection{Multilayer perceptron}

The neurons of an MLP can be arranged into layers with connections feeding forward from one layer to the next, as shown in Figure 1. The first layer is the input layer, where the input data is presented to the network. The last layer is the output layer, where the network produces its prediction data. In between the input and the output layer, there is at least one hidden layer. In each layer, each neuron receives several inputs from the neurons in the previous layer, takes a weighted sum over them, passes it through an activation function and responds with an output. To train the network, a loss function is defined, which is minimised during training by determining the neurons' optimal weights. 
Postprint: Tijskens A., Roels S., Janssen H., 2019. Neural networks for metamodelling the hygrothermal behaviour of building components. Building and Environment 162: 106282

https://doi.org/10.1016/j.buildenv.2019.106282

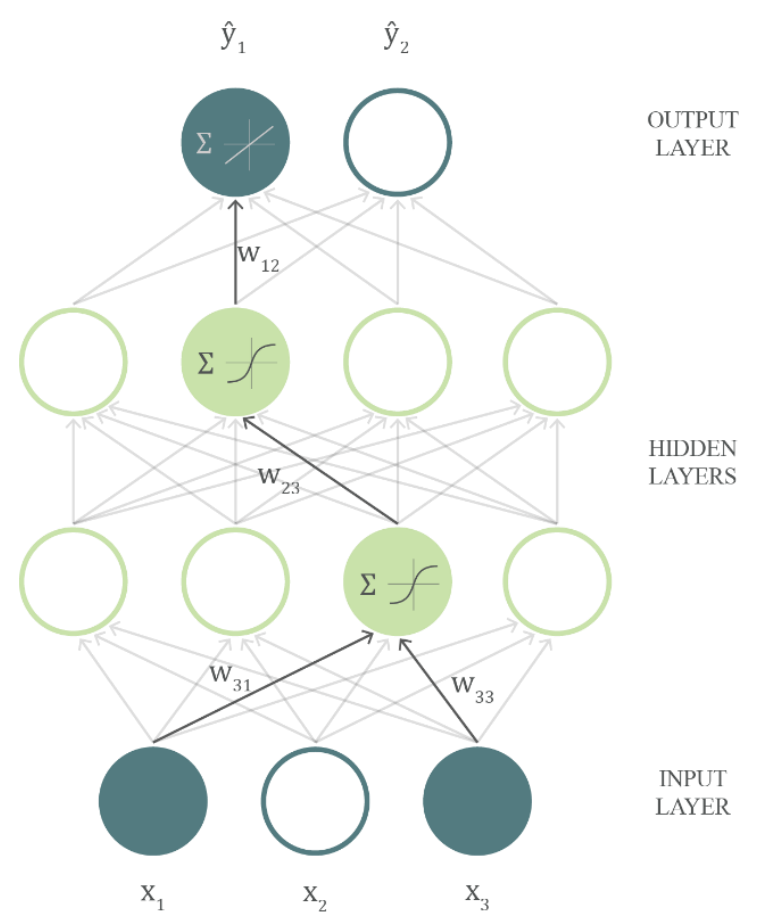

Figure 1: A multilayer perceptron has a feedforward architecture. Each neuron receives inputs from the neurons in the previous layer, takes a weighted sum over them, passes it through an activation function and produces an output.

The input and output layer of the MLP have two dimensions: the number of samples in the dataset and the number of features (input layer) or targets (output layer). A feature is an input parameter, e.g. the exterior temperature. A target is a parameter the network tries to predict, e.g. the temperature at a certain position in the building component. Here, a sample is a single data point, i.e. one time step. Given the dimensionality of the MLP's input and output, the MLP has no notion of sequences or time dependency. In time series prediction, the MLP is sometimes used for predicting the next time step based on the current step [21]. As each step of the sequence is processed independently, the output depends solely on the current input, and not on any past inputs. Hence, the MLP cannot model input-output relations that span multiple time steps. Some MLP applications implicitly capture time by adding a number of previous time steps as additional features, presenting the MLP with a sliding window of context about each data point $[22,23]$. The advantage of this approach is that it is an easy way to incorporate the time-aspect of the dataset, without needing to reach to the more computational expensive networks. The downside is, it fails to capture patterns outside 
Postprint: Tijskens A., Roels S., Janssen H., 2019. Neural networks for metamodelling the hygrothermal behaviour of building components. Building and Environment 162: 106282

https://doi.org/10.1016/j.buildenv.2019.106282

this window and as the window size needs to be determined beforehand, a considerable number of experiments is required to identify the optimum time window. Furthermore, this method only allows small sliding windows, as the dataset memory size increases drastically with the window step size. The performance of the MLP and MLP with a sliding window, applied to predict the hygrothermal response of a massive masonry wall, was investigated in a prior explorative study [24]. It was found that both networks perform adequately for time series that don't require (much) information from the past to predict the present time step, such as the temperature. However, in case of time series with long-term dependencies, such as the relative humidity in a building component, possibly dependent on what happened weeks ago, both networks failed to capture the correct patterns, due to the lack of information about the past. Hence, in this study, the MLP with a sliding window is excluded, while the simple MLP is included for comparison purposes.

\subsection{Recurrent neural networks}

Recurrent neural networks are feedforward neural networks augmented by the inclusion of connections over adjacent time steps, introducing a notion of time into the model. These cyclic connections, called recurrent connections, connect the nodes within a hidden layer across time. Thus, both the input and the output are three-dimensional: the number of samples in the dataset, the number of time steps and the number of features or targets. Here, a sample is one deterministic simulation, containing time series of features and targets. Many varieties of RNN have been proposed, such as Elman networks [25], Jordan networks [26], time delay neural networks [27] and echo state networks [28]. The most successful RNN architectures, however, are the Long Short-Term Memory network (LSTM), developed by Hochreiter and Schmidhuber [29] and the Gated Recurrent Unit (GRU), proposed by Cho et al. [30]. The remainder of this section will hence focus on these two types of recurrent networks. 
Postprint: Tijskens A., Roels S., Janssen H., 2019. Neural networks for metamodelling the hygrothermal behaviour of building components. Building and Environment 162: 106282

https://doi.org/10.1016/j.buildenv.2019.106282

\section{Long Short Term Memory}

The LSTM architecture was first introduced by Hochreiter and Schmidhuber [29] and later improved by Gers et al. [31]. In this architecture, each ordinary neuron in a hidden layer is replaced by a memory cell, shown in

Figure 2.

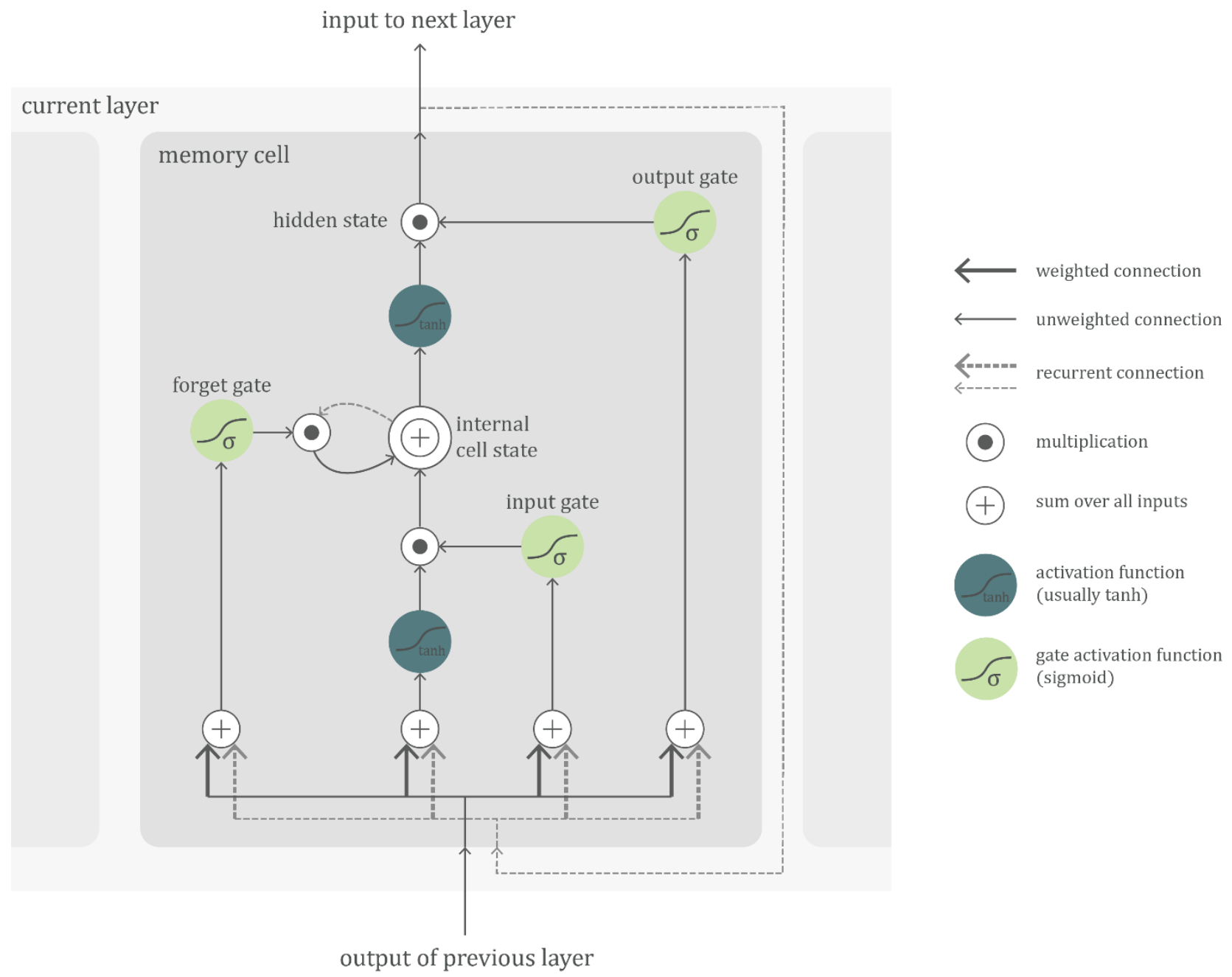

Figure 2: The LSTM cell consists of a cell state, which stores information, and three gates that receive input and control the flow of information.

A memory cell contains a recurrent self-connected node, the 'internal cell state', which allows information to persist across many time steps without being lost, and three multiplicative units, the 'gates', that control the flow of information to the cell state. The gates allow the LSTM cell to store and access the right information over longer periods of time. Each gate receives weighted input from the previous layer at the 
Postprint: Tijskens A., Roels S., Janssen H., 2019. Neural networks for metamodelling the hygrothermal behaviour of building components. Building and Environment 162: 106282

https://doi.org/10.1016/j.buildenv.2019.106282

current time step (represented by the bold solid arrow in Figure 2) and from the current hidden layer at the previous time step, along recurrent connections (bold dashed arrow). The input gate controls the flow of information from new input to the cell state. The cell state stores information from past time steps, while the forget gate controls what information from the cell state in a previous time step is flushed. Finally, the output gate controls what output is produced by the memory cell. This output is called the hidden state.

To understand how information flows through the network across the time dimension, the network can be 'unrolled', as shown in Figure 3. On the left side, a simplified representation of the memory cell is shown, with the recurrent connections. On the right side, the cell is 'unrolled' across time, showing the recurrent connections between the consecutive time steps. By representing the network this way, one can see that each output time step is dependent on the current input, the previous output and the previous internal cell state.

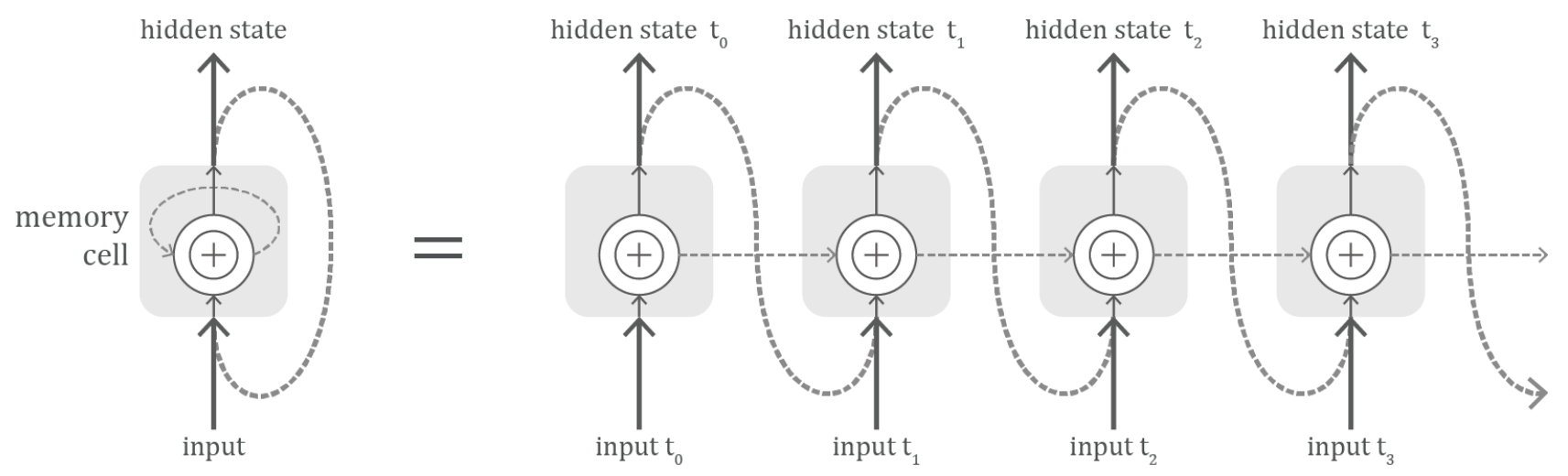

Figure 3: the LSTM network can be 'unrolled' to understand the flow of information through recurrent connections.

Over the past decades, LSTM has proved successful at a range of real-world problems requiring long range memory, such as music generation [31], reinforcement learning [32], speech recognition [33,34], natural language modelling [8], prediction of air pollution concentration [35], financial marketing predictions [36] and many more. 
Postprint: Tijskens A., Roels S., Janssen H., 2019. Neural networks for metamodelling the hygrothermal behaviour of building components. Building and Environment 162: 106282

https://doi.org/10.1016/j.buildenv.2019.106282

\section{Gated Recurrent Unit}

Based on the LSTM architecture, Cho et al. [30] proposed the GRU, shown in Figure 4, which is much simpler to compute and implement. In contrast to the LSTM, the GRU does not have an internal cell state but only produces an output, the hidden state. Additionally, the GRU has only two gates, the reset gate and the update gate, which both receive input from the current input (see bold solid arrows in Figure 4) and the hidden state at the previous time step (bold dashed arrows). The GRU does not have a mechanism to control the degree to which its hidden state is exposed, but exposes the whole state each time. The reset gate allows the previous hidden state to drop any information that is found to be irrelevant later in the future. The update gate controls how much information from the previous hidden state will flow to the current hidden state. As each hidden unit has separate reset and update gates, each hidden unit will learn to capture dependencies over different time scales. The units capturing short-term dependencies will tend to have frequently active reset gates, while the units capturing long-term dependencies will have mostly active update gates. 
Postprint: Tijskens A., Roels S., Janssen H., 2019. Neural networks for metamodelling the hygrothermal behaviour of building components. Building and Environment 162: 106282

https://doi.org/10.1016/j.buildenv.2019.106282

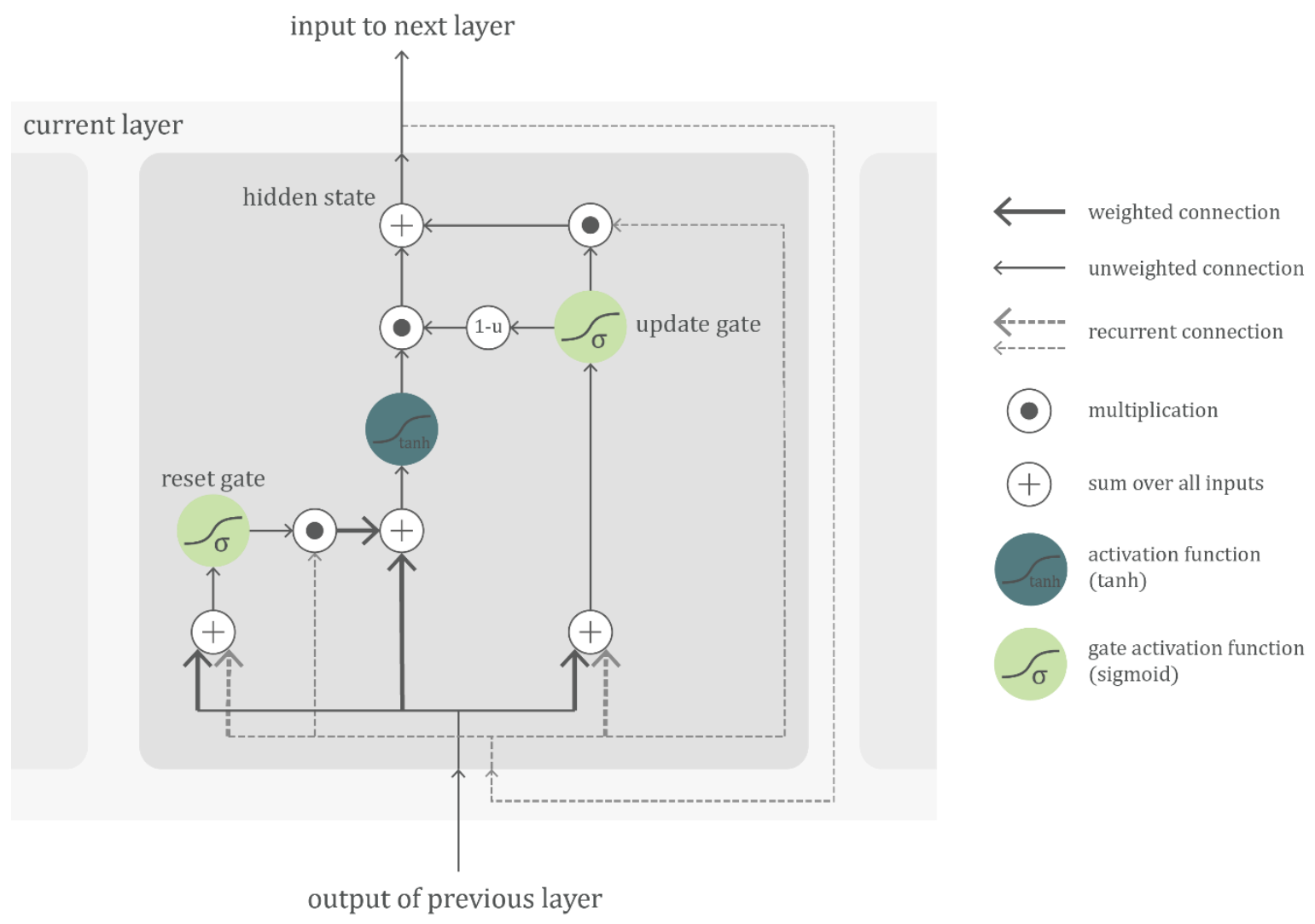

Figure 4: The GRU only has two gates to control the flow of information

The GRU network has proved successful at a range of real-world problems requiring long range memory, such as contextual video recognition [37], financial marketing predictions [38], speech recognition [39] and many more.

The advantage of RNNs is that they automatically learn which information of the past should be kept and which information is redundant. The disadvantage is that each output at a given time step depends on the output at the previous time step, and thus training and predicting is slow. Furthermore, long-term information is easily lost as it needs to be propagated over a lot of time steps; hence, RNN have difficulty dealing with very long time series.

\subsection{Convolutional neural networks}

Convolutional neural networks are a class of deep neural networks most commonly applied to analysing images. The architecture of a CNN is analogous to that of the connectivity pattern of neurons in the human 
Postprint: Tijskens A., Roels S., Janssen H., 2019. Neural networks for metamodelling the hygrothermal behaviour of building components. Building and Environment 162: 106282

https://doi.org/10.1016/j.buildenv.2019.106282

brain, where individual neurons respond to stimuli only in a restricted region of the visual field known as the receptive field. Collections of such fields overlap to cover the entire visual area. The convolution in a CNN copies the response of an individual neuron to visual stimuli. A convolution is a mathematical combination of two functions to produce a third function; it merges two sets of information. In the case of a CNN, the convolution is performed on the input data with the use of a filter to then produce a feature map. The filter slides over the input and at every position, a matrix multiplication is performed. This is repeated for each feature and the result is summed into a new feature map. In case of image processing, the input has four dimensions: samples, width (number of pixels), height (number of pixels) and channels (e.g. three RGB channels). The filter slides over the width and height, for each channel. The size of the filter determines how many input pixels transfer information to the next layer, i.e. the size of the filter determines the size of the receptive field. By using multiple filters, comparable to using multiple neurons in a hidden layer, different feature maps can be learned.

More recently, CNNs have been applied to sequence learning $[11,17,20]$. In this case, the input has three dimensions: samples, time steps and features. Hence, the filter is one-dimensional and slides along the time steps. When working with time series or sequences, usually dilated causal convolutions are used. Causal convolutions ensure that the output of the filter does not depend on future inputs. In a dilated convolution, the filter is applied over an area larger than its length by skipping input values with a certain step. Stacked dilated convolutions enable networks to have very large receptive fields with just a few layers, while preserving the input resolution throughout the network as well as computational efficiency. Often, the dilation factor is exponentially increased for every layer, allowing the receptive field to grow exponentially with network depth. This principle is shown in Figure 5. 
Postprint: Tijskens A., Roels S., Janssen H., 2019. Neural networks for metamodelling the hygrothermal behaviour of building components. Building and Environment 162: 106282

https://doi.org/10.1016/j.buildenv.2019.106282

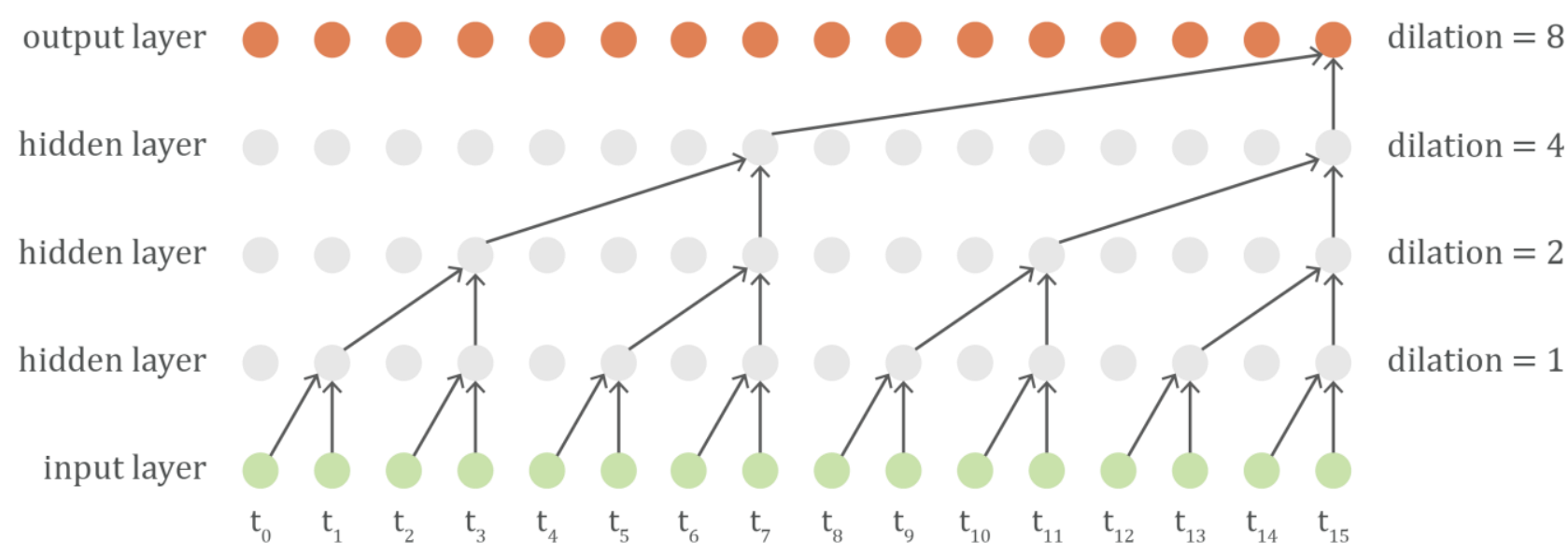

Figure 5: Due to the causal dilated convolutions, an output time step receives information from more input time steps, i.e. the receptive field, with increasing number of hidden layers.

The architecture of the CNN network used in this paper, shown in Figure 6, was based on the Wavenet architecture [11]. Residual blocks with dilated causal convolutions and gated activation units [40] are stacked to create a larger receptive field. Each block has a residual connection [41] to the next block and a parameterised skip connection to the last two layers of the network, both of which speed up convergence and help training deep networks. 
Postprint: Tijskens A., Roels S., Janssen H., 2019. Neural networks for metamodelling the hygrothermal behaviour of building components. Building and Environment 162: 106282

https://doi.org/10.1016/j.buildenv.2019.106282

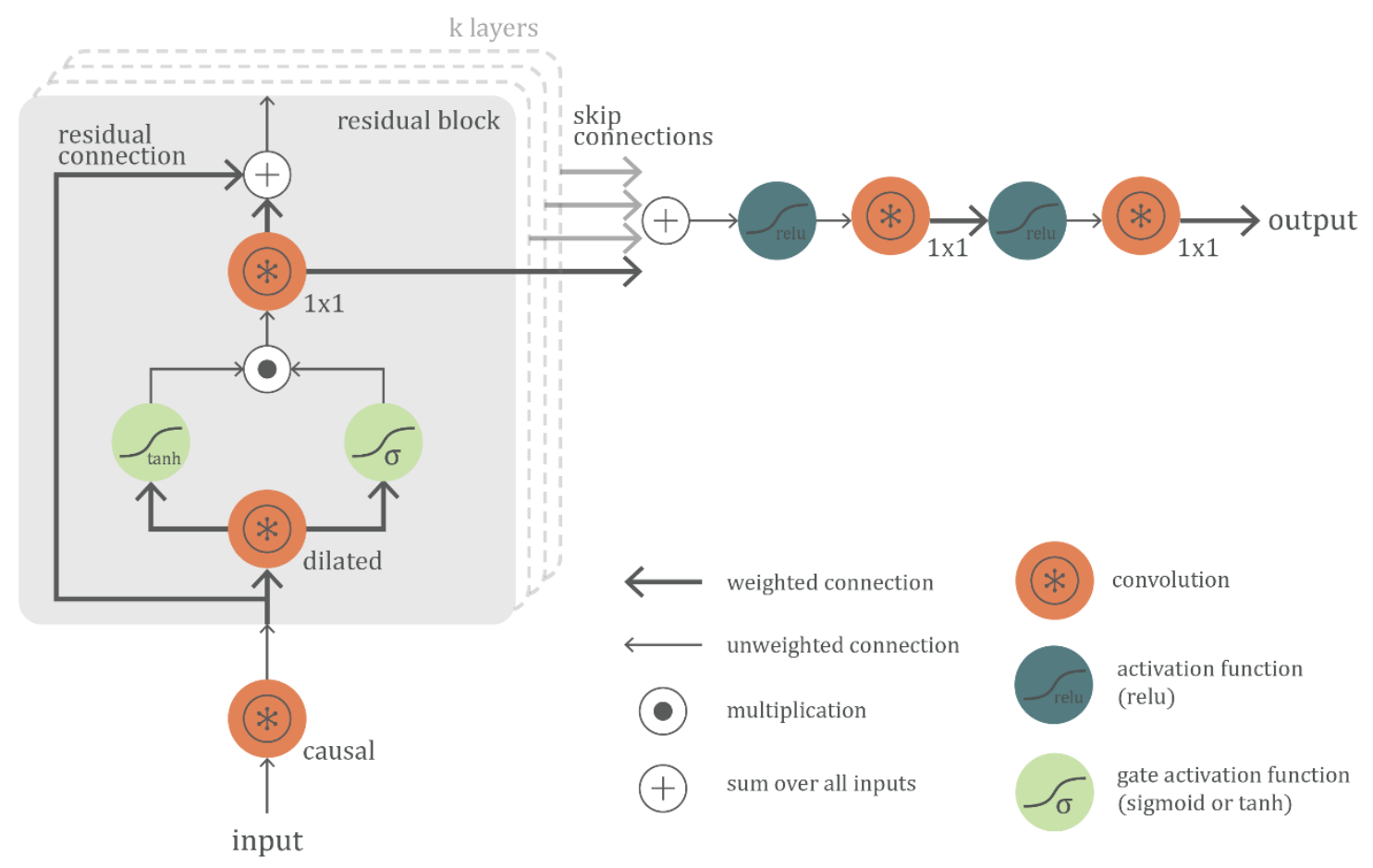

Figure 6: The used CNN architecture with residual blocks and skip connections, based on the Wavenet architecture.

A CNN does not have 'memory' the way the RNN does; the output at a certain time step does not depend on the output on previous time steps, as can be seen in Figure 5. However, thanks to the stacked residual blocks, the CNN has access to a large range of inputs at previous time steps. The advantage of this type of network is that output time steps can be calculated in parallel, which results in a much faster training and predicting compared to the RNN. A disadvantage is that one needs to know how large the receptive field should be when building the network, as information outside the receptive field cannot be used to predict the current time step.

\section{Application Example}

To explore the performance of the different neural networks described above, they are applied to predict the hygrothermal performance of a massive masonry wall in a probabilistic context. In the next section, the 
Postprint: Tijskens A., Roels S., Janssen H., 2019. Neural networks for metamodelling the hygrothermal behaviour of building components. Building and Environment 162: 106282

https://doi.org/10.1016/j.buildenv.2019.106282

calculation object is defined, followed by a section describing the neural networks, of which the prediction performance is discussed in the final section.

\subsection{Calculation object}

This work is part of a European research project on internal insulation for retrofitting historical buildings with massive masonry walls [42]. In this project, the feasibility and durability of applying internal insulation is evaluated by analysing the hygrothermal performance of massive masonry facades before and after insulation through simulations. Because there are many influential but uncertain parameters, a probabilistic approach is applied, which enables taking into account all variability on the exterior climate, geometry, material characteristics, etc. In a probabilistic approach via Monte Carlo, a deterministic simulation is repeated multiple times with different input parameters, thus ensuring a more reliable evaluation. As the uninsulated massive masonry wall is used for comparing hygrothermal performances, this paper uses a massive masonry wall as calculation object to compare the prediction performance of the different neural network types. The massive masonry wall is evaluated as a 1D construction and consists of $30 \mathrm{~cm}$ solid ceramic brick with $1 \mathrm{~cm}$ of plaster as interior finishing. As this is an explorative study, not all influencing probabilistic parameters are taken into account yet, as this allows exploring different network types and architectures more efficiently. The used probabilistic input parameters and their distributions are shown in Table 1. To account for variability in boundary conditions, climate data of four Belgian cities [43], as well as different years of the data and different wall orientations are used. The wind-driven rain load is calculated based on the wall orientation and the exterior climate data. The interior climate is calculated according to European standard EN 15026 [44], based on the exterior climate, and thus includes variability as well. The deterministic boundary conditions are given in Table 2. The characteristics of the used ceramic brick type are given in Table 3 and Figure 7, the latter clearly showing the non-linear aspect of the brick properties. 
Postprint: Tijskens A., Roels S., Janssen H., 2019. Neural networks for metamodelling the hygrothermal behaviour of building components. Building and Environment 162: 106282

\begin{tabular}{|c|c|}
\hline Parameter & Value \\
\hline Exterior climate & D(Gent; Gaasbeek; Oostende, StHubert) \\
\hline Exterior climate start year & $\mathrm{D}(2020 ; 2047)$ \\
\hline $\begin{array}{l}\text { Wall orientation } \\
\text { degrees from North }\end{array}$ & $U(0 ; 360)$ \\
\hline Interior climate [44] & $\mathrm{D}($ load $\mathrm{A}$; load B) \\
\hline
\end{tabular}

Table 1: Input parameter distributions

\begin{tabular}{|c|c|}
\hline Parameter & Value \\
\hline \multicolumn{2}{|l|}{ Exterior surface } \\
\hline Long wave emissivity & 0.9 \\
\hline Solar absorption & 0.4 \\
\hline WDR exposure coefficient & 1 \\
\hline Total heat transfer coefficient $h\left[\mathrm{~W} / \mathrm{m}^{2} \mathrm{~K}\right]$ & 25 \\
\hline Moisture transfer coefficient $\beta[\mathrm{s} / \mathrm{m}]$ & $3 \times 10-8$ \\
\hline \multicolumn{2}{|l|}{ Interior surface } \\
\hline Total heat transfer coefficient $h\left[\mathrm{~W} / \mathrm{m}^{2} \mathrm{~K}\right]$ & 8 \\
\hline Moisture transfer coefficient $\beta[\mathrm{s} / \mathrm{m}]$ & $3 \times 10-8$ \\
\hline \multicolumn{2}{|l|}{ Initial conditions } \\
\hline Initial temperature $\left[{ }^{\circ} \mathrm{C}\right]$ & 20 \\
\hline Initial relative humidity [\%] & 50 \\
\hline \multicolumn{2}{|c|}{ Table 2: Discrete input parameters } \\
\hline Material property & value \\
\hline Dry thermal conductivity $\left(\mathrm{W} / \mathrm{m}^{2} \mathrm{~K}\right)$ & 0.87 \\
\hline Dry vapour resistance factor (-) & 140 \\
\hline Capillary absorption coefficient $\left(\mathrm{kg} / \mathrm{m}^{2} \mathrm{~s}^{0.5}\right)$ & 0.046 \\
\hline Capillary moisture content $\left(\mathrm{m}^{3} / \mathrm{m}^{3}\right)$ & 0.13 \\
\hline Saturation moisture content $\left(\mathrm{m}^{3} / \mathrm{m}^{3}\right)$ & 0.24 \\
\hline
\end{tabular}


Postprint: Tijskens A., Roels S., Janssen H., 2019. Neural networks for metamodelling the hygrothermal behaviour of building components. Building and Environment 162: 106282
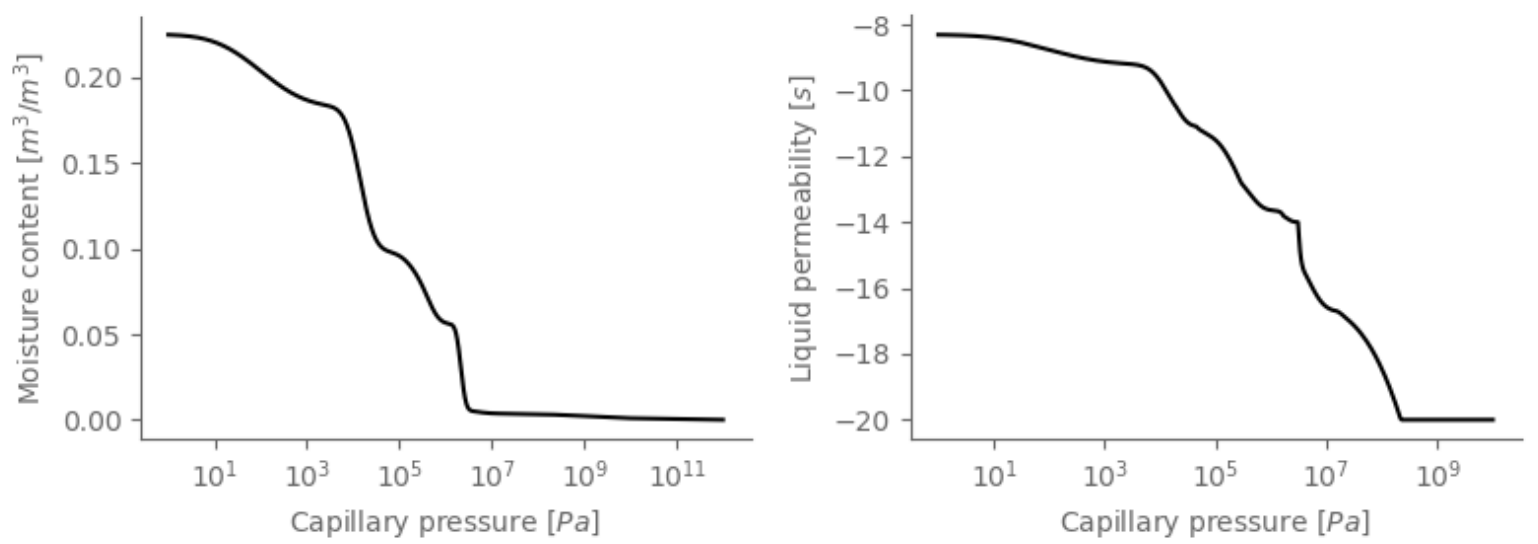

Figure 7: Brick material moisture properties.

Typically, when evaluating the hygrothermal performance of a massive wall, one is interested in frost damage at the exterior surface, wood decay of embedded wooden beam ends and mould growth on the interior surface $[3,6,45,46]$, as shown in Figure 8 . Table 4 gives an overview of the hygrothermal quantities generally required to evaluate these damage patterns, using different damage prediction models such as the number of (moist) freeze-thaw cycles, the VTT Mould Index [47] or the VTT wood decay model [48]. These quantities will be used to evaluate hygrothermal performance and are thus monitored over a period of four years in the simulations. As most damage prediction models require hourly data, and hourly output frequency is used.

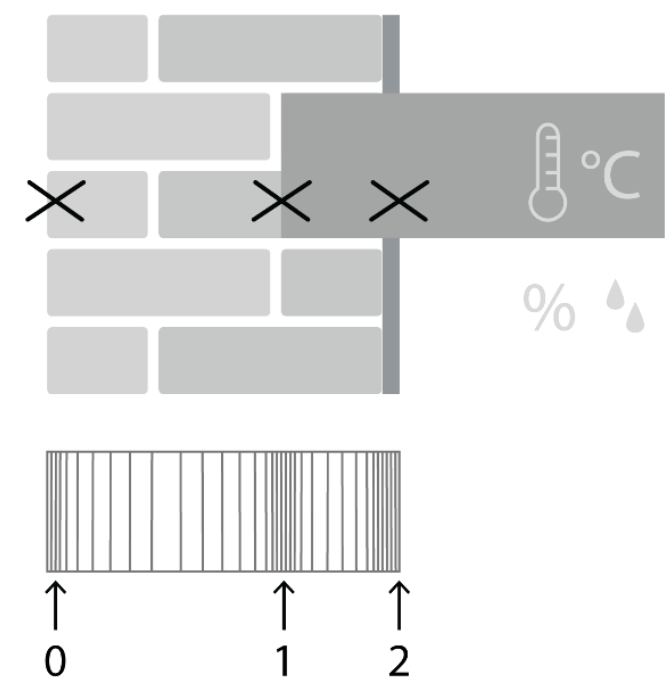

Figure 8: A schematic representation of the 2D building component (top) and the modelled 1D mesh (bottom), with indication of the positions where the hygrothermal performance is monitored. 
Postprint: Tijskens A., Roels S., Janssen H., 2019. Neural networks for metamodelling the hygrothermal behaviour of building components. Building and Environment 162: 106282

https://doi.org/10.1016/j.buildenv.2019.106282

\begin{tabular}{llll}
\multicolumn{1}{c}{ Damage pattern } & Position & Quantities \\
\hline 0 & Frost damage & $0.5 \mathrm{~cm}$ from exterior surface & $\mathrm{T}, \mathrm{RH}, \mathrm{MC}$ \\
1 & Decay of wooden beam ends & $5 \mathrm{~cm}$ from interior brick surface & $\mathrm{T}, \mathrm{RH}$ \\
2 & Mould growth & Interior surface & $\mathrm{T}, \mathrm{RH}$ \\
\hline $\mathrm{T}:$ temperature $\left[{ }^{\circ} \mathrm{C}\right]$ & & \\
$\mathrm{RH}:$ relative humidity $[\%]$ & & \\
$\mathrm{MC}:$ moisture content $\left[\mathrm{kg} / \mathrm{m}^{3}\right]$ & &
\end{tabular}

Table 4: Monitored quantities for the damage patterns at different positions in the wall

To perform a probabilistic evaluation of the massive masonry wall, the input parameters described above are sampled multiple times, using a Sobol sampling scheme [49]. In this study, 80 samples are used. The deterministic model is simulated once for each sampled input parameter combination, using the hygrothermal simulation environment Delphin 5.8 [50]. These 80 simulated samples will form the basis to develop a predictive neural network. This number is chosen based on previous experiments but might not be optimal, i.e. a larger dataset might result in better prediction performance or vice versa, a smaller dataset might provide equally satisfying results.

\subsection{Neural network development}

The first step when developing a predictive neural network is to obtain a 'train' dataset, on which the neural network will be trained, and an independent 'test' dataset, which cannot contain samples from the train set, as it will be used to evaluate the network's performance on unseen data. Thus, the 80 simulated samples, as described in the previous section, are divided into a train set with 64 samples and a test set with 16 samples. In the next step, the neural network is trained by presenting it the input-output combinations of the train set, allowing the network to learn the patterns between input and output. Overfitting is prevented by early stopping, a mechanism that evaluates the performance of the network on the test set during training and stops training if the test set loss increases while the train set performance decreases. The networks are trained to predict hygrothermal time series as given in Table 4, based on the input in Table 1. The inputs are pre-processed to contain only time series, as this simplifies the network architecture. The wall orientation, which is a scalar input parameter, is adopted via the wind-driven rain load and the direct and diffuse short- 
Postprint: Tijskens A., Roels S., Janssen H., 2019. Neural networks for metamodelling the hygrothermal behaviour of building components. Building and Environment 162: 106282

https://doi.org/10.1016/j.buildenv.2019.106282

wave radiation time series. Before presenting the input and output data to the neural networks, all data are standardised (zero mean, unit variance). This ensures that all input features are on the same scale, which allows weighting all features equally in their representation. To ensure errors are penalised equally for all targets, the output data are standardised as well. Once the networks are trained on the train dataset, their performance is evaluated on the test dataset, as this gives an indication of the networks' performance when used as a replacement of the original model.

The architecture of the neural networks is described in section 2. Table 5 shows the used hyperparameters of all network types. These parameters are chosen based on a limited number of experiments and thus might not be optimal. To find the most optimal parameters, a hyperparameter optimization is required, which is time intensive. As this is an explorative study only, this has not been covered yet. Additionally, all metamodels were constructed to have a similar number of trainable parameters, for reliable comparison of training time and performance. Note that, although $10^{6}$ might seem like many parameters, this is not uncommon for neural networks. Due to the highly non-linear nature of the problem, the network requires a lot of parameters to be able to capture the underlying pattern. Furthermore, the amount of data available to train the network largely exceeds the number of trainable parameters, thus the risk for overfitting is small. For each network type, five replications are trained, since different initialisation of the network parameters can lead to different final models.

\begin{tabular}{lccccc} 
NN type & $\begin{array}{c}\text { Hidden } \\
\text { layers }\end{array}$ & $\begin{array}{c}\text { Hidden } \\
\text { neurons }\end{array}$ & $\begin{array}{c}\text { Trainable } \\
\text { parameters }\end{array}$ & Optimizer & $\begin{array}{c}\text { Additional } \\
\text { parameters }\end{array}$ \\
\hline MLP & 5 & 512 & $1.1 \cdot 10^{6}$ & Adam, Ir $=0.002$ & \\
LSTM & 3 & 256 & $1.3 \cdot 10^{6}$ & RMSprop, Ir $=0.002$ & \\
GRU & 3 & 256 & $1.0 \cdot 10^{6}$ & RMSprop, Ir $=0.002$ & \\
CNN & $22^{*}$ & $128^{* *}$ & $1.2 \cdot 10^{6}$ & Adam, Ir $=0.002$ & filter width $=4$ \\
\hline
\end{tabular}

* Two stacks of 11 layers with dilatation factors $\{1,2,4,8, \ldots, 512,1024\}$

** Number of filters in convolutional layers

Table 5: Neural network parameters 
Postprint: Tijskens A., Roels S., Janssen H., 2019. Neural networks for metamodelling the hygrothermal behaviour of building components. Building and Environment 162: 106282

https://doi.org/10.1016/j.buildenv.2019.106282

\subsection{Results and discussion}

The prediction accuracy of the different metamodels is evaluated using three performance indicators: the root mean square error (RMSE), the mean absolute error (MAE), and the coefficient of determination (R2), quantified as follows:

$$
R M S E=\sqrt{\frac{1}{T} \sum(y-\hat{y})^{2}} \quad M A E=\frac{1}{T} \sum|y-\hat{y}| \quad R 2=1-\frac{\sum(y-\hat{y})^{2}}{\sum(y-\bar{y})^{2}} \quad \text { Equation } 1
$$

where $y$ is the true output, $\hat{y}$ is the predicted output, $\bar{y}$ is the mean of the true output and $T$ is the total number of hourly data points. The performance indicators are calculated both for all outputs together as well as for each output separately. The first allows to easily compare the performance of different models, while the latter allows assessing which outputs are more difficult to predict. Additionally, the models' training time is evaluated. 
Postprint: Tijskens A., Roels S., Janssen H., 2019. Neural networks for metamodelling the hygrothermal behaviour of building components. Building and Environment 162: 106282

https://doi.org/10.1016/j.buildenv.2019.106282
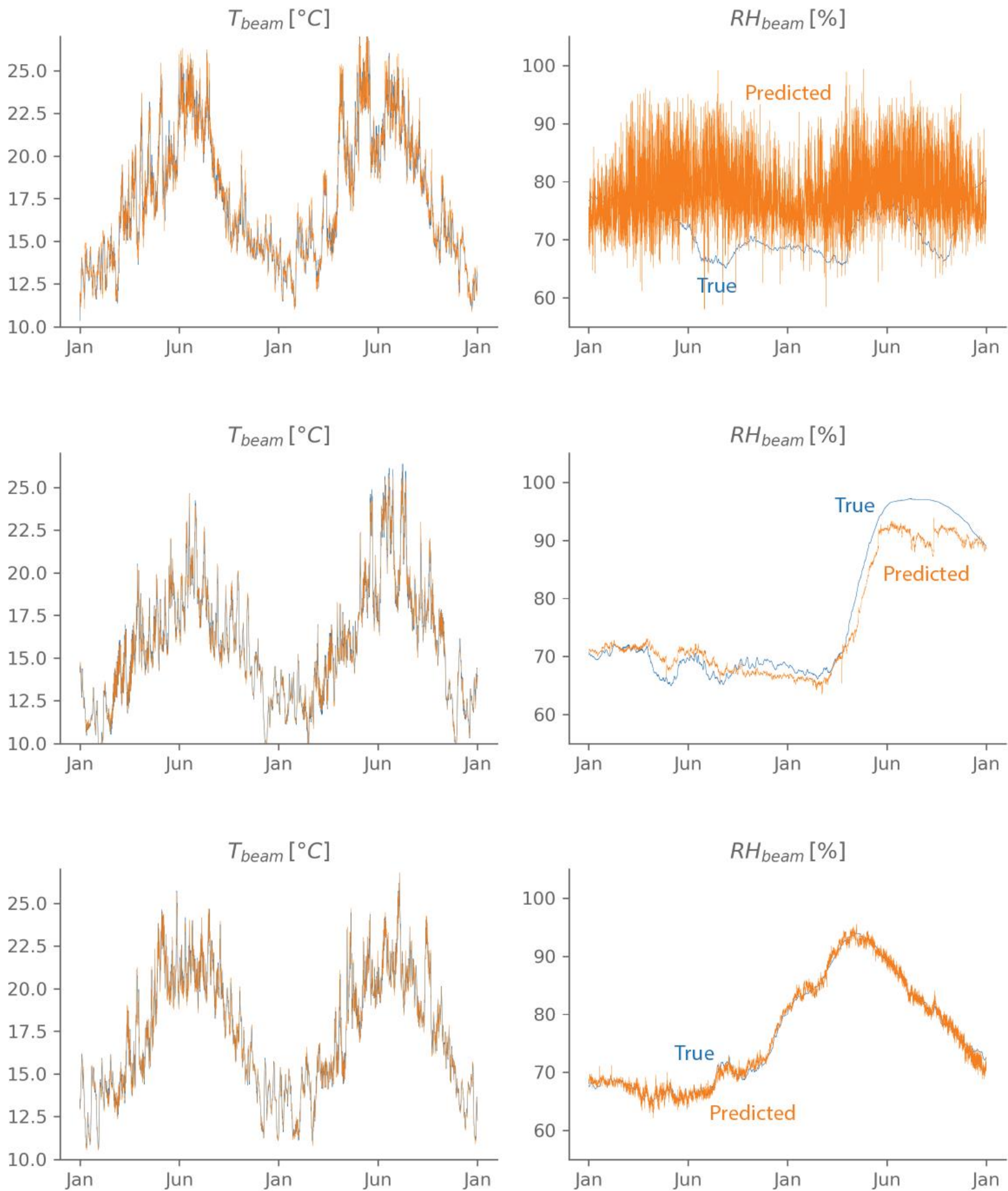

Figure 9: Examples of the predicted temperature and relative humidity course at the position of the wooden beam ends for the MPL (top), LSTM (middle) and CNN (bottom). Note that different samples are plotted for the different network types. The prediction is shown in orange, the true value in blue. 
Postprint: Tijskens A., Roels S., Janssen H., 2019. Neural networks for metamodelling the hygrothermal behaviour of building components. Building and Environment 162: 106282

https://doi.org/10.1016/j.buildenv.2019.106282

Figure 9 shows examples of the predictions for the temperature and relative humidity at the wooden beam ends by the different neural network types. For these predictions, samples from the test set were used. Note that different samples are shown for the different network types, to illustrate the T and RH patterns of different samples. This figure clearly shows that all networks are able to predict the temperature accurately, but only the networks with knowledge about the past can capture the pattern of the relative humidity. Figure 10 shows the performance indicators on the test set as defined by Equation 1, calculated over all outputs, as well as the training time for each neural network type. These results indicate that the MLP is not suited for time series prediction, due to its lack of information on past time steps. The RNNs and CNN on the other hand, perform almost equally well. However, the CNN is a factor 10 faster to train compared to the RNNs. Whereas the RNN models take some 1,5 to 3,5 hours to train the current problem, the CNN models require only 15 to 20 minutes. The prediction time for these networks also differs: it takes 15 seconds for the RNN models to predict 16 samples, whereas it takes only 5 seconds for the CNN model. As a reference, the time required to simulate one sample with the original hygrothermal software is $4-5$ minutes. In a probabilistic setting, where thousands of simulations are required, the extra time spent to develop the neural network is thus largely compensated by the strongly reduced calculation time.

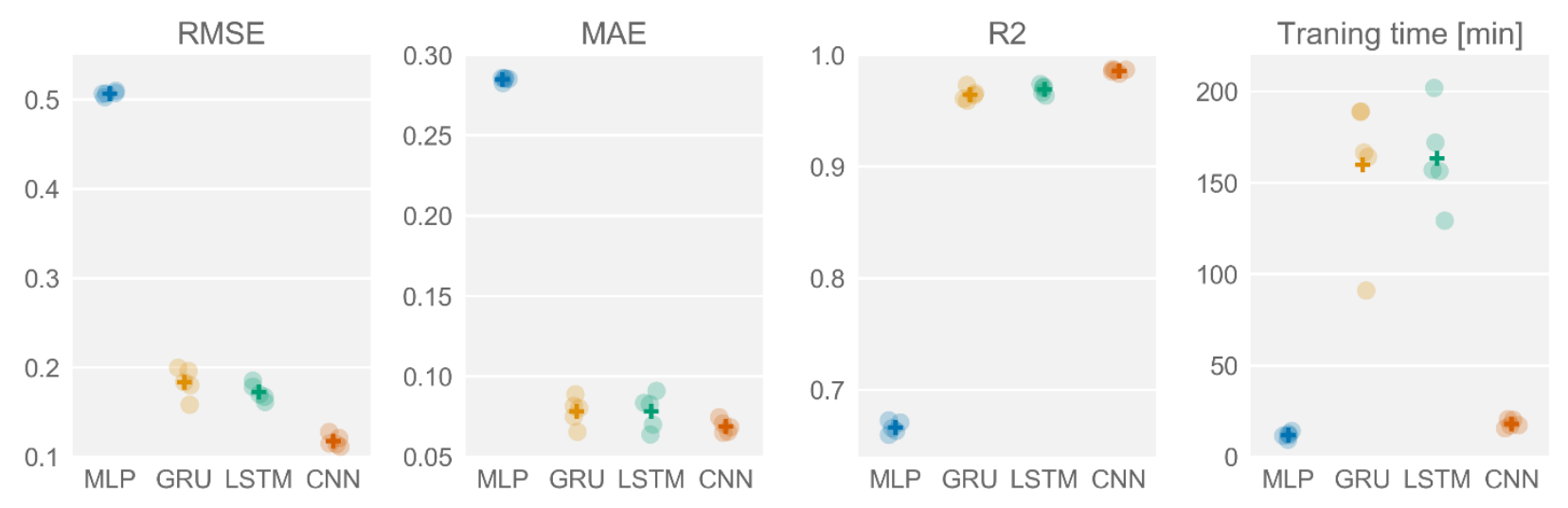

Figure 10: The test set performance indicators, calculated over all outputs together, and training time for each neural network type. The dots show the results of the five replications, while the crosses represent the average result. 
Postprint: Tijskens A., Roels S., Janssen H., 2019. Neural networks for metamodelling the hygrothermal behaviour of building components. Building and Environment 162: 106282

https://doi.org/10.1016/j.buildenv.2019.106282

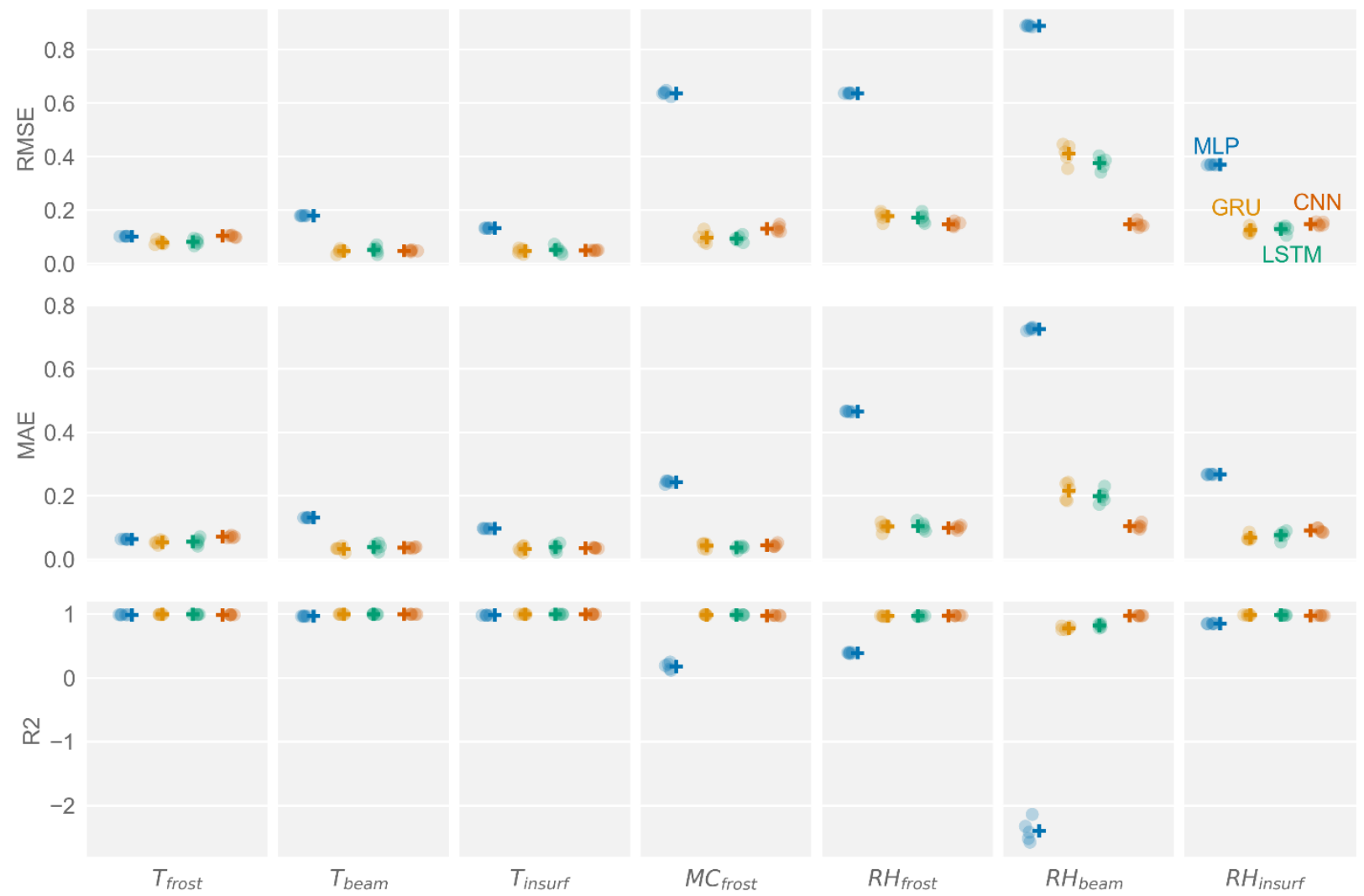

Figure 11: The test set performance indicators, calculated over each output separately for each neural network type. The dots show the results of the five replications, while the crosses represent the average result.

When looking at the performance indicators for each output separately, shown in Figure 11, it is clear that all models are suited to model the temperature profiles. As was already observed in Figure 9, also the MLP performs adequately with respect to the temperatures. Because the wall temperatures responds almost immediately to a change in boundary conditions, not much memory is needed to accurately predict these time series. The relative humidity and moisture content series appear less evident to model. As moisture is transported inwards only slowly, there is a large delay between a change in boundary conditions (e.g. a heavy rain shower) and the response in relative humidity in the wall. At the wooden beam ends, this response delay can go up to several months. The MLP is clearly incapable to capture these long-term temporal dependencies, due to its lack of memory. The RNN and CNN models, on the other hand, are able to capture these complex long-term patterns because of their connections to information from past time-steps. In case of the relative 
Postprint: Tijskens A., Roels S., Janssen H., 2019. Neural networks for metamodelling the hygrothermal behaviour of building components. Building and Environment 162: 106282

https://doi.org/10.1016/j.buildenv.2019.106282

humidity at the wooden beam ends, where the response delay is the largest, the CNN performs significantly better than the RNN models. As explained in section 2.2, RNN models have difficulty learning dependencies from a distant past, because the information needs to persist over a large number of time steps. The CNN on the other hand, has direct access to this long-term information, due to its architecture (see Figure 5). Hence, it is more suited to capture long-term dependencies. This is also visible in Figure 12 and Figure 13, which compare the true output of one test set sample to the output predicted by the LSTM and CNN model, respectively. The residuals (grey) indicate how large the error is between true output (blue) and prediction (orange). Compared to the CNN prediction, the LSTM predicted relative humidity at the beam ends shows larger residuals, and moreover over longer periods. This error is inherent to the LSTM (and GRU) architecture, because each predicted output time step depends on the previous predicted time step(s), which allows errors to accumulate. The CNN has no information on its previous predicted time steps, and thus suffers much less of this problem. However, a different error pattern can be observed in Figure 12; because of this lack of connections to previous predictions, more variability between consecutive output time steps is noted. This causes the predicted time series to be more noisy and explains why the CNN model performs slightly worse when predicting the moisture content or temperature.
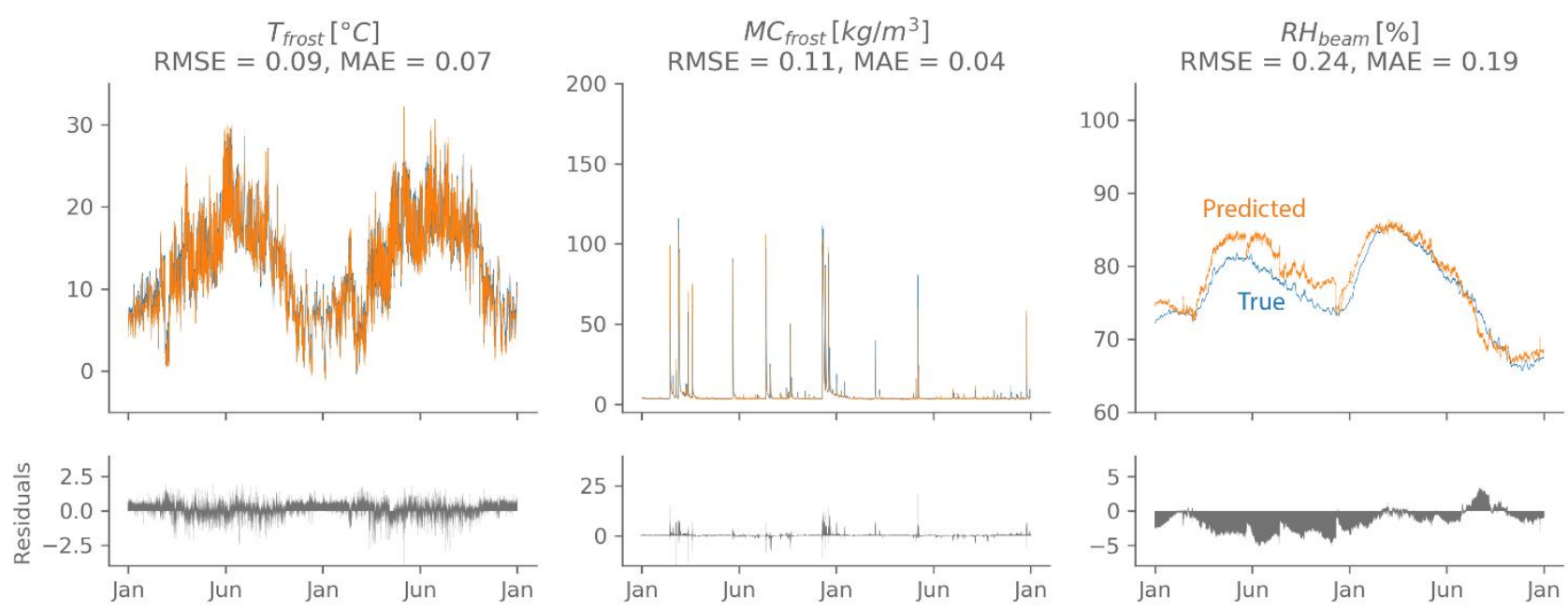

Figure 12: The true output (blue), the output as predicted by the LSTM model (orange) and the residuals (grey). 
Postprint: Tijskens A., Roels S., Janssen H., 2019. Neural networks for metamodelling the hygrothermal behaviour of building components. Building and Environment 162: 106282

https://doi.org/10.1016/j.buildenv.2019.106282
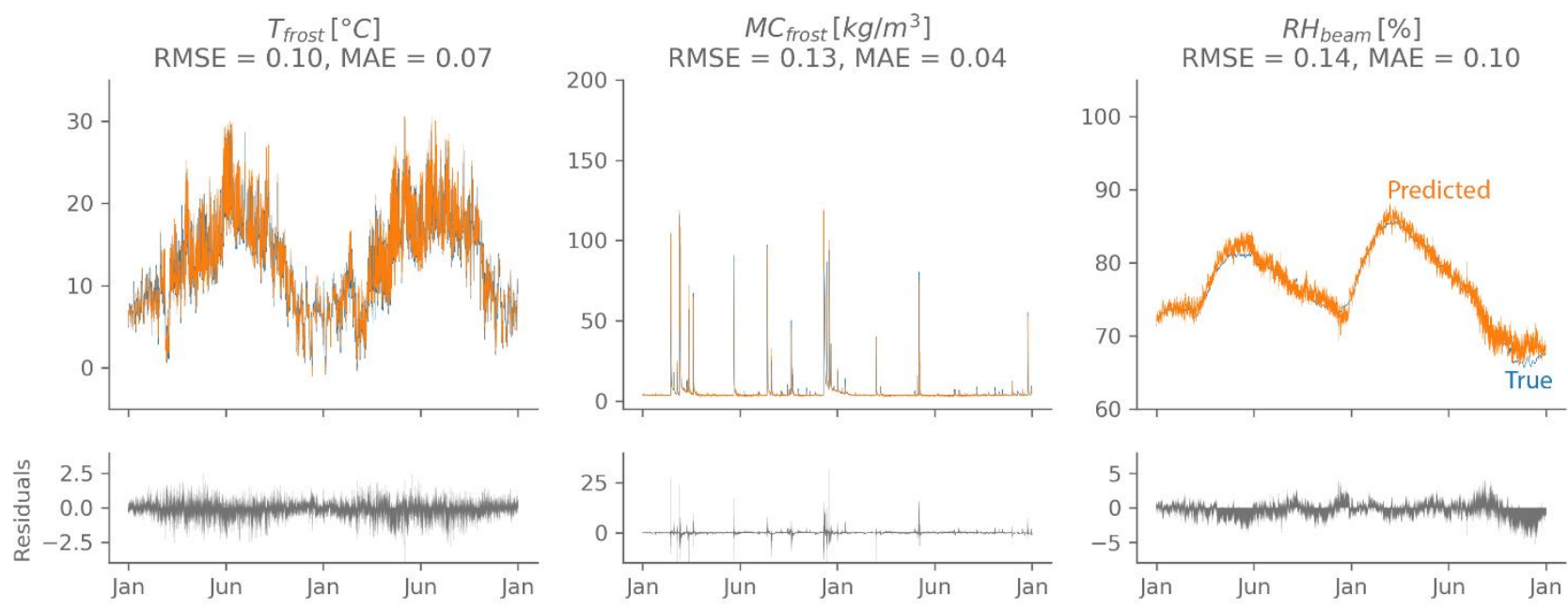

Figure 13: The true output (blue), the output as predicted by the CNN model (orange) and the residuals $\varepsilon_{t}$ (grey).

\section{Conclusion}

This paper proposes the use of fast metamodels to replace heavy hygrothermal simulation models in probabilistic assessments of a building component. As the hygrothermal response of a building component is highly non-linear and transient, this paper opts for neural networks for time series as metamodel, as these have proven successful in many other fields. Three popular types of neural networks are considered: the multilayer perceptron (MLP), recurrent neural networks (RNN), of which the long-short term memory network (LSTM) and gated recurrent unit network (GRU) are included, and the convolutional neural network (CNN). The networks are trained to predict the hygrothermal time series such as the temperature, relative humidity and moisture content at certain positions in a masonry wall, based on the outdoor and indoor climate time series. The MLP has no memory and can only access the current input time step. The RNN's and the CNN both have a memory mechanism, though it works differently for both network types: the RNNs carry information from past input and output time steps through the next one, while the CNN has direct access to multiple past input time steps at once but not to past output time steps. It was found that all network types were capable to predict the temperature accurately. Since the temperature responds almost immediately to 
Postprint: Tijskens A., Roels S., Janssen H., 2019. Neural networks for metamodelling the hygrothermal behaviour of building components. Building and Environment 162: 106282

https://doi.org/10.1016/j.buildenv.2019.106282

a change in boundary conditions, not much memory is needed to capture these patterns. By contrast, only the RNNs and the CNN were able to capture the long-term dependencies needed to predict the relative humidity and moisture content, as these respond much slower to a change in boundary conditions. The CNN performed significantly better at predicting the relative humidity at the wooden beam ends, compared to the RNNs, due to the difference in memory mechanism. Additionally, the CNN is a factor 10 faster to train compared to the RNNs, because its architecture allows processing multiple time steps simultaneously, whereas this happens sequentially in case of the RNNs. To conclude, the CNN appears most suited for the prediction of highly non-linear hygrothermal time series and shows promise to apply as metamodel in more complex probabilistic assessments. Further research is needed to see how the CNN performs when the probabilistic aspect is taken fully into account, i.e. when also scalar and categorical input parameters are included.

\section{ACKNOWLEDGEMENT}

Funding: This work was supported by the European Union's Horizon 2020 research and innovation program under grant agreement No 637268 .

\section{References}

[1] L. Van Gelder, H. Janssen, S. Roels, Probabilistic design and analysis of building performances: Methodology and application example, Energy and Buildings. 79 (2014) 202-211. doi:10.1016/j.enbuild.2014.04.042.

[2] H. Janssen, S. Roels, L. Van Gelder, Annex 55 Reliability of Energy Efficient Building Retrofitting Probability Assessment of Performance \& Cost (RAP-RETRO), 2013.

[3] E. Vereecken, L. Van Gelder, H. Janssen, S. Roels, Interior insulation for wall retrofitting - A probabilistic analysis of energy savings and hygrothermal risks, Energy and Buildings. 89 (2015) 231244. doi:10.1016/j.enbuild.2014.12.031.

[4] H. Janssen, Monte-Carlo based uncertainty analysis: Sampling efficiency and sampling convergence, Reliability Engineering \& System Safety. 109 (2013) 123-132. doi:10.1016/j.ress.2012.08.003.

[5] L. Van Gelder, P. Das, H. Janssen, S. Roels, Comparative study of metamodelling techniques in building energy simulation: Guidelines for practitioners, Simulation Modelling Practice and Theory. 49 (2014) 245-257. doi:10.1016/j.simpat.2014.10.004.

[6] V. Marincioni, G. Marra, H. Altamirano-medina, Development of predictive models for the probabilistic moisture risk assessment of internal wall insulation, Building and Environment. 137 
Postprint: Tijskens A., Roels S., Janssen H., 2019. Neural networks for metamodelling the hygrothermal behaviour of building components. Building and Environment 162: 106282

https://doi.org/10.1016/j.buildenv.2019.106282

(2018) 257-267. doi:10.1016/j.buildenv.2018.04.001.

[7] T. Mikolov, M. Karafiat, L. Burget, J.H. Cernock, S. Khudanpur, Recurrent neural network based language model, in: INTERSPEECH 2010, Makuhari, Chiba, Japan, 2010: pp. 1045-1048. doi:10.1007/978-94-007-2564-5.

[8] M. Sundermeyer, R. Schluter, H. Ney, LSTM Neural Networks for Language Modeling, in: INTERSPEECH 2012, Portland, OR, USA, 2012: pp. 194-197.

[9] M. Elbayad, L. Besacier, J. Verbeek, Pervasive Attention: 2D Convolutional Neural Networks for Sequence-to-Sequence Prediction, 2018. http://arxiv.org/abs/1808.03867.

[10] J. Gehring, M. Auli, D. Grangier, D. Yarats, Y.N. Dauphin, Convolutional Sequence to Sequence Learning, 2017. doi:10.1002/nt.2620030507.

[11] A. van den Oord, S. Dieleman, H. Zen, K. Simonyan, O. Vinyals, A. Graves, N. Kalchbrenner, A. Senior, K. Kavukcuoglu, WaveNet: A Generative Model for Raw Audio, (2016) 1-15. doi:10.1109/ICASSP.2009.4960364.

[12] A. Van Den Oord, Y. Li, I. Babuschkin, K. Simonyan, O. Vinyals, K. Kavukcuoglu, G. Van Den Driessche, E. Lockhart, L.C. Cobo, F. Stimberg, N. Casagrande, D. Grewe, S. Noury, S. Dieleman, E. Elsen, N. Kalchbrenner, H. Zen, A. Graves, H. King, T. Walters, D. Belov, D. Hassabis, Parallel WaveNet: Fast HighFidelity Speech Synthesis, (2017). http://arxiv.org/abs/1711.10433.

[13] A. Karpathy, G. Toderici, S. Shetty, T. Leung, R. Sukthankar, L. Fei-Fei, Large-scale Video Classification with Convolutional Neural Networks, in: Conference on Computer Vision and Pattern Recognition, Columbus, Ohio, USA, 2014: pp. 1725-1732. doi:10.1109/CVPR.2014.223.

[14] G. Giacinto, F. Roli, Design of effective neural network ensembles for image classification purposes, 19 (2000) 699-707. doi:10.1016/S0262-8856(01)00045-2.

[15] K. Gregor, I. Danihelka, A. Graves, D.J. Rezende, D. Wierstra, DRAW: A Recurrent Neural Network For Image Generation, 2015. doi:10.1038/nature14236.

[16] C. Dong, C.C. Loy, K. He, X. Tang, Learning a Deep Convolutional Network for Image Super-Resolution, in: D. Fleet, T. Pajdla, B. Schiele, T. Tuytelaars (Eds.), Computer Vision -- ECCV 2014, Springer International Publishing, Cham, 2014: pp. 184-199.

[17] A. Borovykh, S. Bohte, C.W. Oosterlee, Conditional Time Series Forecasting with Convolutional Neural Networks, (2017) 1-22. http://arxiv.org/abs/1703.04691.

[18] J.D. Velásquez-Henao, S. Gutiérrez, C.J. Franco, Using a Dynamic Artificial Neural Network for Forecasting the Volatility of a Financial Time Series, Revista Ingenierías Universidad de Medellín. 12 (2013) 127-136.

[19] W. Groß, S. Lange, J. Bödecker, M. Blum, Predicting Time Series with Space-Time Convolutional and Recurrent Neural Networks, in: European Symposium on Artificial Neural Networks, Bruges (Belgium), 2017: pp. 26-28. https://www.elen.ucl.ac.be/Proceedings/esann/esannpdf/es2017-38.pdf.

[20] S. Bai, J.Z. Kolter, V. Koltun, An Empirical Evaluation of Generic Convolutional and Recurrent Networks for Sequence Modeling, (2018). http://arxiv.org/abs/1803.01271.

[21] M. Soleimani-Mohseni, B. Thomas, P. Fahlén, Estimation of operative temperature in buildings using artificial neural networks, Energy and Buildings. 38 (2006) 635-640. doi:10.1016/j.enbuild.2005.10.004.

[22] M. Cottrell, B. Girard, Y. Girard, M. Mangeas, C. Muller, Neural modeling for time series: a statistical stepwise method for weight eliminationfeedforward networks are universal approximators, IEEE Transactions on Neural Networks. 6 (1995) 1355-1364. 
Postprint: Tijskens A., Roels S., Janssen H., 2019. Neural networks for metamodelling the hygrothermal behaviour of building components. Building and Environment 162: 106282

https://doi.org/10.1016/j.buildenv.2019.106282

[23] L. Mba, P. Meukam, A. Kemajou, Application of artificial neural network for predicting hourly indoor air temperature and relative humidity in modern building in humid region, Energy and Buildings. 121 (2016) 32-42. doi:10.1016/j.enbuild.2016.03.046.

[24] A. Tijskens, S. Roels, H. Janssen, Neural networks to predict the hygrothermal response of building components in a probabilistic framework, in: Conference Proceedings of IBPC, Syracuse, NY, USA, 2018.

[25] J.L. Elman, Finding structure in time, Cognitive Science. 14 (1990) 179-211. doi:10.1016/03640213(90)90002-E.

[26] M.I. Jordan, Attractor Dynamics and Parallelism in a Connectionist Sequential Machine, IEEE Computer Society Neural Networks Technology Series. (1990) 112-127.

[27] K.J. Lang, A.H. Waibel, G.E. Hinton, A time-delay neural network architecture for isolated word recognition, Neural Networks. 3 (1990) 23-43. doi:10.1016/0893-6080(90)90044-L.

[28] H. Jaeger, The "Echo State" Approach to Analysing and Training Recurrent Neural Networks, 2001.

[29] S. Hochreiter, J. Urgen Schmidhuber, Long Short-term Memroy, Neural Computation. 9 (1997) 17351780. doi:10.1162/neco.1997.9.8.1735.

[30] K. Cho, B. van Merrienboer, C. Gulcehre, D. Bahdanau, F. Bougares, H. Schwenk, Y. Bengio, Learning Phrase Representations using RNN Encoder-Decoder for Statistical Machine Translation, (2014). doi:10.3115/v1/D14-1179.

[31] F.A. Gers, D. Eck, J. Schmidhuber, Applying LSTM to Time Series Predictable through Time-Window Approaches, in: ICANN, 2001: pp. 669-676.

[32] B. Bakker, Reinforcement Learning Memory, in: International Conference on Neural Information Processing Systems, 2002: pp. 1475-1482. https://papers.nips.cc/paper/1953-reinforcementlearning-with-long-short-term-memory.pdf.

[33] A. Graves, J. Schmidhuber, Framewise phoneme classification with bidirectional LSTM and other neural network architectures, Neural Networks. 18 (2005) 602-610. doi:10.1016/j.neunet.2005.06.042.

[34] A. Graves, S. Fernandez, F. Gomez, J. Schmidhuber, Connectionist Temporal Classification: Labelling Unsegmented Sequence Data with Recurrent Neural Networks, in: International Conference on Machine Learning, Pittsburgh, PA, USA, 2006: pp. 369-376. doi:10.1145/1143844.1143891.

[35] X. Li, L. Peng, X. Yao, S. Cui, Y. Hu, C. You, T. Chi, Long short-term memory neural network for air pollutant concentration predictions: Method development and evaluation, Environmental Pollution. 231 (2017) 997-1004. doi:10.1016/j.envpol.2017.08.114.

[36] T. Fischer, C. Krauss, Deep learning with long short-term memory networks for financial market predictions, European Journal of Operational Research. 270 (2018) 654-669. doi:10.1016/j.ejor.2017.11.054.

[37] M. Jung, H. Lee, J. Tani, Adaptive detrending to accelerate convolutional gated recurrent unit training for contextual video recognition, Neural Networks. 105 (2018) 356-370. doi:10.1016/j.neunet.2018.05.009.

[38] G. Shen, Q. Tan, H. Zhang, P. Zeng, J. Xu, Deep Learning with Gated Recurrent Unit Networks for Financial Sequence Predictions, in: International Congress of Information and Communication Technology, Elsevier B.V., 2018: pp. 895-903. doi:10.1016/j.procs.2018.04.298.

[39] M. Ravanelli, P. Brake, M. Omologo, Y. Bengio, Improving speech recognition by revising gated recurrent units, Proceedings of the Annual Conference of the International Speech Communication 
Postprint: Tijskens A., Roels S., Janssen H., 2019. Neural networks for metamodelling the hygrothermal behaviour of building components. Building and Environment 162: 106282

https://doi.org/10.1016/j.buildenv.2019.106282

Association, INTERSPEECH. (2017) 1308-1312. doi:10.21437/Interspeech.2017-775.

[40] A. van den Oord, N. Kalchbrenner, O. Vinyals, L. Espeholt, A. Graves, K. Kavukcuoglu, Conditional Image Generation with PixelCNN Decoders, (2016). doi:10.1007/s109995-010-0626-3.

[41] K. He, X. Zhang, S. Ren, J. Sun, Deep Residual Learning for Image Recognition, 19 (2015) 107-117. http://arxiv.org/abs/1512.03385.

[42] Robust Internal Thermal Insulation of Historic Buildings, (n.d.). https://www.ribuild.eu/ (accessed May 7, 2019).

[43] European Commission, Climate for Culture: damage risk assessment, economic impact and mitigation strategies for sustainable preservation of cultural heritage in times of climate change, 2014.

[44] European committee for Standardisation, EN 15026:2007 - Hygrothermal performance of building components and building elements - Assessment of moisture transfer by numerical simulation, 2007.

[45] M. Harrestrup, S. Svendsen, Internal insulation applied in heritage multi-storey buildings with wooden beams embedded in solid masonry brick façades, Building and Environment. 99 (2016) 59-72. doi:10.1016/j.buildenv.2016.01.019.

[46] X. Zhou, D. Derome, J. Carmeliet, Hygrothermal modeling and evaluation of freeze-thaw damage risk of masonry walls retrofitted with internal insulation, Building and Environment. (2017). doi:10.1016/j.buildenv.2017.08.001.

[47] H. Viitanen, Development of an improved model for mould growth: Modelling, in: Proceedings of the 8th Symposium on Building Physics in the Nordic Countries NSB2008, Denmark, Copenhagen, 2008.

[48] H. Viitanen, T. Toratti, L. Makkonen, Development of Service Life Model for Wooden Structures, in: International Conférence On Durability of Building Materials and Components, 2011: pp. 1-8.

[49] T. Hou, D. Nuyens, S. Roels, H. Janssen, Quasi-Monte-Carlo-based probabilistic assessment of wall heat loss, Energy Procedia. 132 (2017) 705-710. doi:10.1016/j.egypro.2017.10.010.

[50] Delphin 5.8 [Computer software], (2014). https://www.bauklimatik-dresden.de/delphin/index.php. 